hep-th/0304199

\title{
Negative Energy Density in Calabi-Yau Compactifications
}

\author{
Thomas Hertog ${ }^{1}$, Gary T. Horowitz ${ }^{1}$, and Kengo Maeda ${ }^{2}$ \\ ${ }^{1}$ Department of Physics, UCSB, Santa Barbara, CA 93106 \\ hertog@vulcan.physics.ucsb.edu, gary@physics.ucsb.edu \\ ${ }^{2}$ Yukawa Institute for Theoretical Physics, Kyoto University, Kyoto 606-8502, Japan \\ kmaeda@yukawa.kyoto-u.ac.jp
}

\begin{abstract}
We show that a large class of supersymmetric compactifications, including all simply connected Calabi-Yau and $G_{2}$ manifolds, have classical configurations with negative energy density as seen from four dimensions. In fact, the energy density can be arbitrarily negative - it is unbounded from below. Nevertheless, positive energy theorems show that the total ADM energy remains positive. Physical consequences of the negative energy density include new thermal instabilities, and possible violations of cosmic censorship.
\end{abstract}




\section{Introduction}

Standard supersymmetric compactifications of string theory consist of solutions of the form $M_{4} \times K$ where $M_{4}$ is four dimensional Minkowski spacetime and $K$ is a compact, Ricci flat manifold admitting a covariantly constant spinor. Familiar examples of $K$ include $T^{n}, K 3$, Calabi-Yau spaces, and manifolds with $G_{2}$ holonomy. It turns out that there is an important qualitative difference between some of these supersymmetric compactifications and others. We will show that a large class, including all simply connected Calabi-Yau and $G_{2}$ manifolds, have the surprising property that there are configurations with negative energy density. In other words, from a four dimensional perspective, there can be finite regions of space with negative energy. In fact, the energy density is unbounded from below! In contrast, these properties do not hold at least not in the same way - for $T^{n}$ or $K 3$ compactifications.

We will work in the context of classical vacuum solutions to higher dimensional general relativity. So there is no matter or energy density in the higher dimensional space. The negative energy arises from the Kaluza-Klein compactification. These results clearly apply to string theory since vacuum solutions are all (approximate) solutions to string theory and $M$ theory with the other fields set to zero. We should emphasize that the negative energy regions we have in mind are very different from previous discussions of negative energy in Kaluza-Klein theory [1, 2]. In those cases, supersymmetry was broken and the spacetime was not topologically a product $\mathbf{R}^{4} \times K$. In contrast, the compactifications we consider here are all supersymmetric and the spacetime will be a product manifold.

The key mathematical fact which allows configurations of negative energy density is the following. As we review in section 2, all simply connected compact manifolds of dimension five, six, or seven admit Riemannian metrics with positive scalar curvature. Other manifolds, such as $T^{n}$ and $K 3$ do not. Positive scalar curvature on $K$ leads to negative energy density as follows. Vacuum solutions can be characterized by their initial data on $\mathbf{R}^{\mathbf{3}} \times K$. Since we want to minimize the energy, we set the time derivatives of the metric to zero. For time symmetric initial data, the Einstein constraint equations reduce to the vanishing of the scalar curvature, $\mathcal{R}=0$. For a product metric on $\mathbf{R}^{3} \times K, \mathcal{R}=\mathcal{R}_{3}+\mathcal{R}_{K}$ where $\mathcal{R}_{3}$ is the scalar curvature on $\mathbf{R}^{3}$ and $\mathcal{R}_{K}$ is the scalar curvature on $K$. If $\mathcal{R}_{K}>0$, we must take $\mathcal{R}_{3}<0$. 
But negative scalar curvature on $\mathbf{R}^{\mathbf{3}}$ is just like negative energy density. (Recall that the usual constraint of $3+1$ dimensional general relativity says ${ }^{1} \mathcal{R}_{3}=2 \rho$ in the time symmetric case.) Therefore, from an effective four dimensional standpoint, positive scalar curvature on $K$ acts like negative energy density. In other words, ten dimensional vacuum gravity has configurations with effective negative energy density! Of course, we must require that the metric on $K$ approaches the standard Ricci flat metric at infinity, so we cannot keep the metric a product everywhere. However, one can satisfy this boundary condition and keep the region of negative energy density by taking the metric to be product inside a large ball of radius $R_{0}$. In a finite transition region, $R_{0}<r<R_{1}$, one can change the metric on $K$ to the standard Ricci flat metric.

Not only is there negative energy density in four dimensions, but this energy can be arbitrarily negative. This follows immediately from the fact that there is no upper bound on the scalar curvature $\mathcal{R}_{K}$. Given a metric on $K$ with $\mathcal{R}_{K}>0$, one can clearly rescale it by a constant factor and make the scalar curvature arbitrarily large. This shows that the negative energy density is unbounded from below. (Of course, once the curvature becomes larger than the string scale, there may be significant $\alpha^{\prime}$ corrections.)

As we discuss in section 2, the metrics with positive scalar curvature on $K$ lie a finite distance away from the moduli space of Ricci flat metrics. This shows that the negative energy is a nonperturbative effect, which cannot be seen at any finite order in perturbation theory about the moduli space. In terms of an effective four dimensional potential, we will see that (part of) the negative energy density can be described by a scalar field with a negative potential which falls off exponentially. Negative potentials are familiar in supersymmetric AdS compactifications, but to our knowledge, this is the first time they have been seen in supersymmetric asymptotically flat compactifications.

Given the existence of negative energy density, it is natural to ask if the total ADM energy can be negative. Recall that the total energy for any spacetime which asymptotically approaches $M_{4} \times K$ is well-defined. In the construction outlined above, the negative energy grows like the volume of the ball. The transition region includes positive energy, but one might expect this only grows like the area so the total energy

\footnotetext{
${ }^{1}$ We set $8 \pi G=1$.
} 
could be negative. This would imply that these supersymmetric compactifications are unstable. However, this does not occur. There are positive energy theorems $[3,4,5]$ which ensure that the total energy remains positive. Coleman and De Luccia [6] showed long ago that gravity can stabilize a false vacuum. (This was applied to supersymmetric vacua in $[7,8]$.) They considered a scalar field with a potential with two local minima that are close together. We have discovered an extreme generalization of this phenomenon: gravity is stabilizing a false vacuum in a theory with a potential which is unbounded from below. This is a flat space analog of the familiar situation for supersymmetric AdS vacua $[9,10]$.

Despite the fact that the total energy remains positive, the existence of regions of negative energy does have physical consequences which we begin to explore. These include new instabilities at finite temperature, and possible violations of cosmic censorship.

An outline of this paper is as follows. In section 2 we review the mathematical results about the existence of metrics of positive scalar curvature that we will need. The next section shows how to use these results to construct configurations with negative energy density. It also gives a qualitative discussion of the effective four dimensional potential that results from compactification. To gain intuition for how the total energy can remain positive given the unbounded negative potential, we discuss a simple model of four dimensional gravity coupled to a single scalar field in section 4 . The following section reviews the positive energy theorems which guarantee that the ADM energy is never negative. In section 6 we discuss some of the physical consequences of the negative energy density. The final section contains remarks about other applications of our results, e.g., to the stability of de Sitter spacetime, and using S-duality to obtain negative energy density even in K3 compactifications.

\section{Metrics with positive scalar curvature}

Since metrics with positive scalar curvature play such an important role in our construction, we review here some of the main mathematical results that we will need. (For a more complete discussion of results up to 1989, see [11].) Let $K$ be a compact manifold. At first sight, finding a metric whose scalar curvature is positive at every point on $K$ sounds easy, since it is only one scalar inequality on the entire metric. 
Indeed, every compact $K$ (of dimension $n>2$ ) admits metrics with negative scalar curvature. However it is known that there is a topological obstruction to the existence of metrics with positive scalar curvature. For example, the torus $T^{n}$ (for any $n$ ) does not admit any metric with ${ }^{2} \mathcal{R}>0$ [12]. The same is true for $K 3$. In this case the proof is easy: The index for the Dirac operator on $K 3$ is nonzero. This means that for every metric on $K 3$, there are nonzero spinors which solve the Dirac equation: $\gamma^{i} \nabla_{i} \epsilon=0$. However, if we square the Dirac operator we get

$$
\nabla^{2} \epsilon-\frac{1}{4} \mathcal{R} \epsilon=0 .
$$

Multiplying by the adjoint spinor and integrating over $K$ yields

$$
\int|\nabla \epsilon|^{2}+\frac{1}{4} \mathcal{R}|\epsilon|^{2}=0 .
$$

This shows that the metric cannot have positive scalar curvature. This argument clearly applies to any manifold for which the index of the Dirac operator, $\hat{A}(K)$, is nonzero.

There is a generalization of $\hat{A}(K)$ which has been shown to completely characterize when a (simply connected, spin) manifold admits a metric of positive scalar curvature. It is usually denoted $\alpha(K)$, and was first introduced in 1974 by Hitchen [13] who showed that if there is a metric with $\mathcal{R}>0$, then $\alpha(K)=0$. The converse was established by Stolz in 1990 [14]: If $\alpha(K)=0$, there always exists a metric with $\mathcal{R}>0$. If the dimension of the manifold is a multiple of four, $\alpha(K)$ is proportional to $\hat{A}(K)$. In general, the definition of $\alpha(K)$ is more involved, but for our purposes, its most important property is that it can be nonzero only in dimensions $n=0,1,2,4 \bmod 8$. (This assumes $n>4$.) So there is a possible obstruction to positive scalar curvature only in these dimensions. Even in these dimensions, this obstruction only applies to spin manifolds: If $K$ does not admit spinors, then it always admits a metric of positive scalar curvature [15]. Taken together, these results show the following:

Every simply connected manifold of dimension 5, 6, or 7 admits a metric with positive scalar curvature.

The situation for nonsimply connected manifolds is much more complicated and still not well understood [16]. We have already remarked that the torus does not

\footnotetext{
${ }^{2}$ In this section we will only consider the scalar curvature of $K$ and drop the subscript $K$ on $\mathcal{R}$.
} 
admit a metric with $\mathcal{R}>0$ even in 5,6,7 dimensions. However, it has been shown that if $K$ is simply connected and admits a metric of positive scalar curvature, then $K / Z_{p}$ also admits a metric of positive scalar curvature if $p=2$ or an odd integer [17]. It is likely that all six dimensional Calabi-Yau spaces (whether simply connected or not) admit metrics of positive scalar curvature [18].

Let $K$ be a compact $n$ dimensional manifold admitting metrics of positive scalar curvature, and consider the space of all smooth Riemannian metrics on $K$. There is the following simple description of this space. First note that the space of all metrics is connected: Any metric can be continuously deformed into any other metric. This is most easily seen by characterizing each metric at a point by an orthonormal frame, and noting that one set of linearly independent vectors can certainly be continuously connected to another. Second, under a conformal rescaling $\tilde{g}_{m n}=\psi^{4 /(n-2)} g_{m n}$ the scalar curvature transforms as

$$
\tilde{\mathcal{R}}=\psi^{-4 /(n-2)}\left[\mathcal{R}-a \psi^{-1} \nabla^{2} \psi\right]
$$

where $a=4(n-1) /(n-2)$. Now consider the lowest eigenvalue of the conformally invariant Laplacian:

$$
-a \nabla^{2} \psi+\mathcal{R} \psi=\lambda_{0} \psi
$$

The corresponding eigenfunction is nonzero and can be used as a conformal factor in (2.3). The resulting scalar curvature has the same sign as the eigenvalue $\lambda_{0}$. Although this construction does not yield constant scalar curvature, the Yamabe conjecture and subsequent proof [19] shows that one can always conformally rescale these metrics to ones with constant scalar curvature. This is very useful since $\lambda_{0}$ is just a function on the space of metrics and divides it up into two regions depending on whether the metric can be rescaled to positive scalar curvature or not.

By continuity, the boundary of the region with $\mathcal{R}>0$ contains metrics with $\mathcal{R}=0$. In addition, there is the possibility of "islands of $\mathcal{R}=0$ " metrics a finite distance away from the positive scalar curvature metrics (The subspace of zero scalar curvature metrics need not be connected). However, those metrics must be Ricci flat, $\mathcal{R}_{m n}=0$. To see this, note that if $\mathcal{R}_{m n} \neq 0$ we can perturb the metric by a multiple of the Ricci tensor, $\delta g_{m n}=b \mathcal{R}_{m n}$. Since $\mathcal{R}_{m n}$ is traceless and divergence free, the change in the scalar curvature under this perturbation is $\delta \mathcal{R}=-b \mathcal{R}_{m n} \mathcal{R}^{m n}$. So by changing the sign of the coefficient $b$, we can construct nearby metrics with either 
positive or negative scalar curvature. This shows that any metric with zero scalar curvature but nonzero Ricci tensor, must lie on a boundary between positive and negative $\mathcal{R}$ metrics. ${ }^{3}$

- Ricci flat metrics

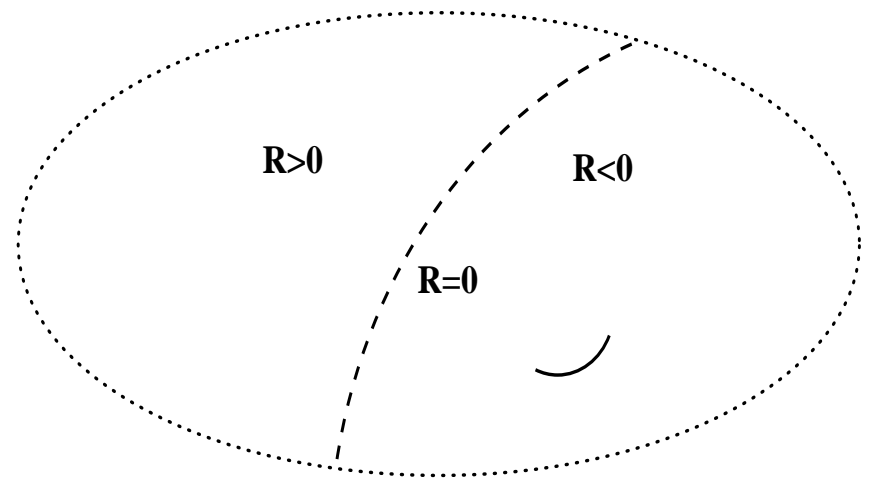

Figure 1: The space of metrics for $K$ is shown, where $K$ is e.g. a simply connected Calabi-Yau or $G_{2}$ manifold. The moduli space of Ricci-flat metrics is surrounded by metrics that can be conformally rescaled to have $\mathcal{R}<0$.

A Ricci flat metric could also lie on the boundary of a region of $\mathcal{R}>0$ metrics. So a natural question to ask is: Where is the moduli space of Ricci flat metrics in the usual supersymmetric compactification? We now show that they must lie a finite distance away from the positive scalar curvature metrics (see Fig. 1). Supersymmetry guarantees that there are no tachyons in the perturbative spectrum. However all Ricci flat metrics which can be perturbed to both positive and negative scalar curvature must have tachyons. This can be seen as follows. A general perturbation can be divided into a pure trace, and transverse traceless pieces. A pure trace perturbation produces a first order change in the scalar curvature proportional to $\nabla^{2} h$. This integrates to zero so it clearly cannot have definite sign. A transverse traceless perturbation, $h_{m n}$, does not change $\mathcal{R}$ to first order. To study its second order effects, consider the functional

$$
S=\int_{K} \mathcal{R} \sqrt{g}
$$

\footnotetext{
${ }^{3}$ This is one way to see that the only metrics on $K 3$ with zero scalar curvature are the Ricci flat metrics.
} 
Its first variation is

$$
\delta S=\int_{K}\left(\mathcal{R}_{m n}-\frac{1}{2} \mathcal{R} g_{m n}\right) h^{m n} \sqrt{g}
$$

This vanishes for all perturbations since the background is Ricci flat. The second variation is

$$
\delta^{2} S=\int_{K} h^{m n} \Delta_{L} h_{m n} \sqrt{g}
$$

where $\Delta_{L}$ is the Lichnerowicz operator coming from the linearized Einstein equation. It is now clear that $\Delta_{L}$ must have both positive and negative eigenvalues in order for $\delta^{2} S$ to have either sign. But this means that if we start with the product spacetime $M_{4} \times K$ with metric $d s^{2}=\eta_{\mu \nu} d x^{\mu} d x^{\nu}+g_{m n}(y) d y^{m} d y^{n}$, some metric perturbations of the form $\phi(x) h_{m n}(y)$ will be tachyonic. Thus the moduli space of supersymmetric, Ricci flat metrics must lie a finite distance ${ }^{4}$ away from the metrics with $\mathcal{R}>0$, as shown in Figure 1.

\section{$3 \quad$ Negative Energy Density in Four Dimensions}

As described in the introduction, the existence of metrics of positive scalar curvature on $K$ leads to effective negative energy density in the four dimensional theory. In this section, we explain this in more detail and describe some qualitative features of the four dimensional effective potentials which arise.

We want to consider vacuum solutions which asymptotically approach $M_{4} \times K$, where $K$ is, e.g., a simply-connected Calabi-Yau space. We can characterize these solutions in terms of initial data. To minimize the mass, we will consider time symmetric initial data which are spherically symmetric on $\mathbf{R}^{\mathbf{3}}$. There are many ways to construct solutions to the initial value constraint $\mathcal{R}=0$ which have regions of negative energy density on $\mathbf{R}^{\mathbf{3}}$. We now describe one approach.

Consider the metric

$$
d s^{2}=\left(1-\frac{2 m(r)}{r}\right)^{-1} d r^{2}+r^{2} d \Omega_{2}^{2}+g_{m n}(r, y) d y^{m} d y^{n}
$$

\footnotetext{
${ }^{4}$ There probably exist manifolds $K$ that admit Ricci flat metrics on the boundary of an $\mathcal{R}>0$ region, but they are necessarily not supersymmetric.
} 
where the indices $m, n$ label the extra compact dimensions. The metric $g_{m n}(r, y)$ denotes a one parameter family of metrics on $K$. The Ricci scalar of (3.1) is

$$
\begin{aligned}
\mathcal{R}= & -\left(1-\frac{2 m(r)}{r}\right)\left[-\frac{1}{4} \partial_{r} g^{m n} \partial_{r} g_{m n}+g^{\prime \prime}+\frac{2}{r} g^{\prime}+\frac{1}{4}\left(g^{\prime}\right)^{2}\right] \\
& +\mathcal{R}_{K}+\partial_{r} m\left(\frac{4}{r^{2}}+\frac{g^{\prime}}{r}\right)-\frac{m}{r^{2}} g^{\prime}
\end{aligned}
$$

where $g^{\prime} \equiv g^{m n} \partial_{r} g_{m n}, g^{\prime \prime}=\partial_{r} g^{\prime}$, and $\mathcal{R}_{K}$ is the scalar curvature of $g_{m n}(r, y)$ at fixed $r$.

Inside some region $r<R_{0}$, we choose $g_{m n}(r, y)$ to be independent of $r$, and equal to some metric with $\mathcal{R}_{K}=2 V_{0}$, a positive constant. In this case, the constraint $\mathcal{R}=0$ reduces to $\partial_{r} m=-V_{0} r^{2} / 2$ which is easily solved for $m(r)$ yielding a region of constant negative energy density. We now pick a radius $R_{1}>R_{0}$ and choose any path in the space of metrics which connects our positive scalar curvature metric $g_{m n}\left(R_{0}, y\right)$ to a metric on the moduli space, $g_{m n}\left(R_{1}, y\right)$. In general, we cannot solve $\mathcal{R}=0$ for $m(r)$ because there is nontrivial $y$ dependence. However, we can find an $m(r)$ so that $\mathcal{R} \geq 0$. We can either view this as nonvacuum initial data for string theory, by adding say a dilaton with $\varphi=0$ and $\dot{\varphi}^{2}=\mathcal{R}$, or we can obtain vacuum initial data by a subsequent conformal rescaling of the nine dimensional metric (3.1). Let $\widetilde{d s}^{2}=e^{4 \psi / 7} d s^{2}$, then the change in the scalar curvature is given by (2.3). So if $\psi$ is a solution to the conformally invariant Laplace equation in nine dimensions

$$
-\nabla^{2} \psi+\frac{7}{32} \mathcal{R} \psi=0
$$

then the rescaled scalar curvature vanishes. In order for the rescaled metric to be nonsingular and asymptotically flat, we need a solution $\psi$ which is nonvanishing and goes to one at infinity. One can show that such solutions always exist when $\mathcal{R} \geq 0$ [20]. This conformal rescaling can only decrease the total energy since the ADM mass changes by

$$
\Delta M \propto-\oint \nabla \psi \propto-\int \mathcal{R} \psi<0 .
$$

From the four dimensional viewpoint, the metric on $K$ is like an (infinite) collection of scalar fields with potential $-\mathcal{R}_{K}$. Qualitatively, this potential has a local minimum at zero when $g_{m n}$ is on the moduli space. There is then a finite positive barrier separating this minimum from a region where the potential is negative. Since 
the potential is just the scalar curvature, the height of the barrier is roughly $1 / L_{K}^{2}$ where $L_{K}$ is a characteristic size of $K$. Thus large Calabi-Yau spaces have small potential barriers. The width of the potential is harder to estimate since it depends on mathematical details about the space of metrics on $K$ which are not yet known. For example, a key open question is: How close does the moduli space of Ricci flat metrics come to the region of positive scalar curvature metrics? The positive energy theorems we discuss later can be used to give some information about this distance.

Once one reaches a metric of constant scalar curvature $\mathcal{R}_{K}=2 V_{0}>0$, one can always rescale the metric by a conformal factor which is constant on $K$, to increase the curvature and make the effective three dimensional energy density more negative. We can easily compute the effective potential for this mode. Let us start with a product metric on $\mathbf{R}^{\mathbf{3}} \times K, d s^{2}=d s_{3}^{2}+d s_{K}^{2}$. Let $\phi$ be a function depending only on $\mathbf{R}^{3}$. The scalar curvature of the metric

$$
d s^{2}=e^{-n \phi} d s_{3}^{2}+e^{2 \phi} d s_{K}^{2}
$$

is

$$
\mathcal{R}=e^{n \phi}\left[\mathcal{R}_{3}+2 V_{0} e^{-(2+n) \phi}-\frac{n(n+2)}{2}(\nabla \phi)^{2}\right]
$$

where $n$ is the dimension of $K$. The second term on the right is just the scalar curvature of the rescaled metric on $K$. The vacuum constraint is $\mathcal{R}=0$, and in $3+1$ dimensions, the energy density is $\rho=\mathcal{R}_{3} / 2$. So we obtain

$$
\rho=\frac{n(n+2)}{4}(\nabla \phi)^{2}-V_{0} e^{-(2+n) \phi} .
$$

Rescaling $\phi$ to have a standard kinetic term we get

$$
\rho=\frac{1}{2}(\nabla \tilde{\phi})^{2}-V_{0} e^{-\alpha \tilde{\phi}}
$$

where

$$
\alpha^{2}=\frac{2(n+2)}{n} .
$$

So the potential is not only negative, but falls off exponentially fast. Notice that for more than one extra dimension, $2<\alpha^{2}<6$.

There are other ways to achieve large $\mathcal{R}_{K}$ (and hence large negative energy density). We mentioned in the last section that one can always conformally rescale a 
metric on $K$ to one with constant scalar curvature whose sign depends on the lowest eigenvalue, $\lambda_{0}$, of the conformal Laplacian. If $\lambda_{0} \leq 0$, the rescaled metric is unique for fixed volume. In other words, the only remaining freedom is the constant rescalings discussed above. However, if $\lambda_{0}>0$, the conformally related metric with constant positive curvature is generically not unique, even when the volume is fixed. There is a maximum value of the scalar curvature in each conformal equivalence class, but it can be arbitrarily big in nearby conformal classes. More precisely, given a conformal class with $\lambda_{0}>0$, for any $\varepsilon>0$ and any large number $N$ there is another conformal class within $\varepsilon$ of the first (in a $C^{0}$ topology) with a metric of unit volume and constant scalar curvature larger than $N$ [21]. This surprising result shows that the maximum scalar curvature in each conformal class is not a continuous function ${ }^{5}$.

There is clearly a lot of freedom in the construction of configurations with negative energy density. One can choose any path in the space of metrics from the moduli space to the region with $\mathcal{R}_{K}>0$, and then choose any path in the region of positive scalar curvature metrics to make $\mathcal{R}_{K}$ large. One approach is to divide the motion up into nonconformal motion and conformal rescalings. For the nonconformal motion, there are two natural choices: One is to choose a path that only includes metrics of constant scalar curvature and fixed volume; the other is to keep a local volume element fixed. This second option greatly simplifies (3.2) since $g^{\prime}=0$, but since the scalar curvature does not remain constant, one needs to rescale the final metric by a position dependent conformal factor to obtain constant $\mathcal{R}_{K}>0$.

\section{$4 \quad$ Scalar Field Model}

In the next section we will review the theorems which show that despite the unbounded negative energy density, the total energy must remain positive. To gain intuition into how this is possible, we now consider a simple model of four dimensional gravity coupled to a single scalar field with potential $V(\phi)$. To model the situation we have for the compactifications, we consider potentials $V(\phi)$ which are negative in a region of $\phi$ which is separated by a positive barrier from a local minimum at $\phi_{f}>0$ where the potential vanishes. We consider configurations where $\phi \rightarrow \phi_{f}$

\footnotetext{
${ }^{5}$ This depends on the topology one puts on the space of conformal classes. In a stronger topology, this function will probably be continuous [22].
} 
asymptotically, and ask whether the total energy can be negative. In the absence of gravity, the answer is clearly yes: Inside a large ball of radius $R_{0}$, one can keep $\phi$

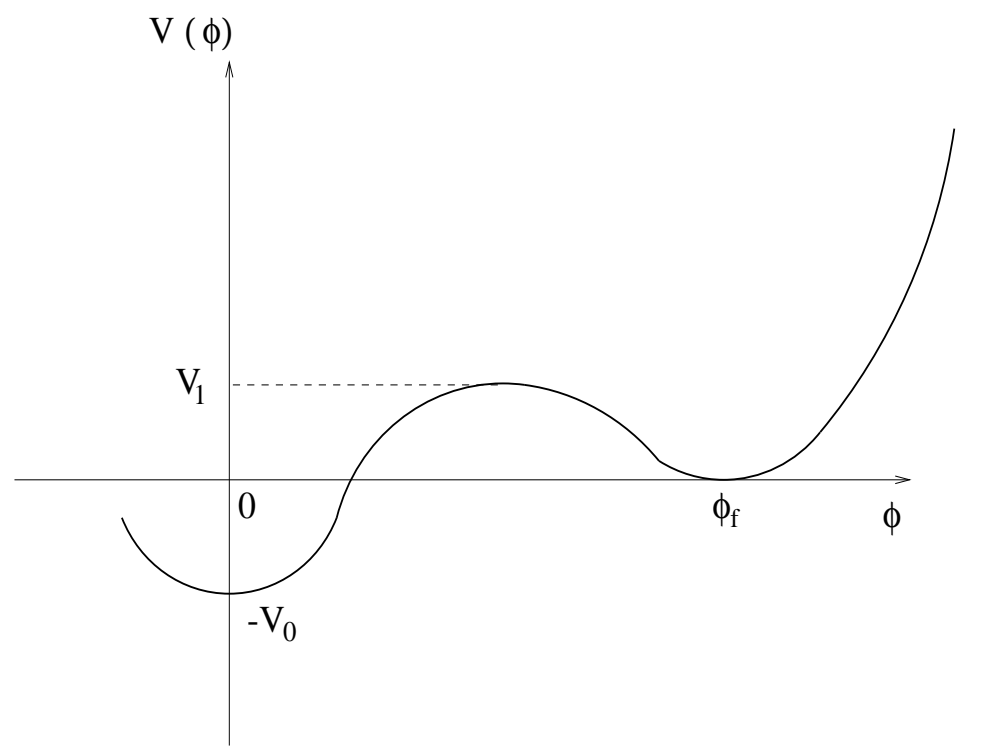

Figure 2: A potential with a negative minimum at $\phi=0$ that is separated by a positive barrier from a local minimum at $\phi_{f}$.

constant at a value where the potential is negative, and then have $\phi$ approach $\phi_{f}$ in a transition region. Since the negative energy in the ball grows like the volume and the positive energy in the transition region only grows like the area, the total energy can clearly be negative. However, as first shown by Coleman and De Luccia [6], gravity can modify this picture.

Since we want to minimize the energy, we set all the time derivatives to zero. For time symmetric initial data the constraint equations reduce to

$$
{ }^{(3)} \mathcal{R}=2 \rho
$$

where

$$
\rho=\frac{1}{2} g^{i j} \phi_{, i} \phi_{, j}+V(\phi) .
$$

Since spatial gradients raise the energy, we consider a spherically symmetric configuration with metric

$$
d s^{2}=\left(1-\frac{2 m(r)}{r}\right)^{-1} d r^{2}+r^{2} d \Omega_{2}^{2}
$$


The constraint then yields the following equation for the "mass" $m$ as a function of the radius,

$$
m^{\prime}+\frac{1}{2} m r \phi^{\prime 2}=\frac{1}{2} r^{2}\left[V(\phi)+\frac{1}{2} \phi^{\prime 2}\right]
$$

where prime denotes the ordinary derivative with respect to $r$. The ADM mass is simply the value of $m(r)$ at infinity.

We can choose $\phi(r)$ arbitrarily and solve (4.4) for $m(r)$. The general solution is

$$
2 m(r)=e^{-\int_{0}^{r} \hat{r} \phi^{\prime 2} / 2 d \hat{r}} \int_{0}^{r} e^{\int_{0}^{\tilde{r}} \hat{r} \phi^{\prime 2} / 2 d \hat{r}}\left[V(\phi)+\frac{1}{2} \phi^{\prime 2}\right] \tilde{r}^{2} d \tilde{r}
$$

Consider now a potential with a negative minimum at $\phi=0$ that is separated by a positive barrier from a local minimum at $\phi_{f}$, where the potential vanishes. We call the height of the positive barrier $V_{1}$, and the depth of the negative minimum $V(0)=-V_{0}$ (see Fig. 2). We pick two radii $R_{1}>R_{0}$ and set $\phi=0$ for $r<R_{0}, \phi=\phi_{f}$ for $r>R_{1}$, and let $\phi$ be any smooth increasing function for $R_{0}<r<R_{1}$. The solution for $m(r)$ for this configuration is

$$
2 m(r)=e^{-\int_{R_{0}}^{r} \hat{r} \phi^{\prime 2} / 2}\left[-\frac{1}{3} V_{0} R_{0}^{3}+\int_{R_{0}}^{r}\left(V(\phi)+\frac{1}{2} \phi^{\prime 2}\right) e^{\int_{R_{0}}^{\tilde{r}} \hat{r} \phi^{\prime 2} / 2} \tilde{r}^{2} d \tilde{r}\right] .
$$

One sees that the positive energy in the barrier is enhanced relative to the negative energy by an exponential factor. We shall see that the ADM mass can nevertheless be negative provided that $V_{0} / V_{1}$ is sufficiently large. To identify the approximate criteria for this to be the case, we minimize the exponential factor by taking

$$
\phi(r)=\mu \ln \left(r / R_{0}\right)
$$

in the transition region. This fixes the relation between $R_{0}$ and $R_{1}$ to be $R_{1}=R_{0} e^{\phi_{f} / \mu}$. The ADM mass $M=m(\infty)=m\left(R_{1}\right)$ is given by

$$
\begin{aligned}
2 M= & e^{-\mu \phi_{f} / 2}\left[-\frac{1}{3} V_{0} R_{0}^{3}+\frac{\mu^{2} R_{0}}{\left(\mu^{2}+2\right)}\left(e^{\phi_{f}\left(\mu^{2}+2\right) / 2 \mu}-1\right)\right. \\
& \left.+\frac{R_{0}^{3}}{\mu} \int_{0}^{\phi_{f}} e^{x\left(\mu^{2}+6\right) / 2 \mu} V(x) d x\right] .
\end{aligned}
$$

Notice that the last term, coming from the transition region, scales like $R_{0}^{3}$, just like the first term. If the slope $\mu$ and the width of the potential barrier $\phi_{f}$ are $\sim \mathcal{O}(1)$, then the integral is $\sim V_{1}$. For $R_{0} \gg 1$, the condition for the ADM mass $M$ to be 
negative is then simply $V_{0}>V_{1}$. For an arbitrary slope $\mu$ and width $\phi_{f}$, and assuming $V(\phi)=V_{1}$ for $0<\phi<\phi_{f}$, we have $M<0$ if (again for $R_{0} \gg 1$ )

$$
\frac{V_{0}}{V_{1}}>\frac{6}{\left(\mu^{2}+6\right)}\left(e^{\phi_{f}\left(\mu^{2}+6\right) / 2 \mu}-1\right) .
$$

The right hand side is minimized for $\mu$ of order one.

Intuitively, one reason that it is harder to get negative total energy in theories with gravity is that a constant negative energy density produces a hyperbolic space with constant negative curvature. In this space, the volume inside a ball of large radius is equal to the volume of a thin shell at this radius with a width equal to the scale of curvature.

As we saw in the last section, the potentials which arise from Calabi-Yau compactifications are unbounded from below. Now suppose we modify the potential for

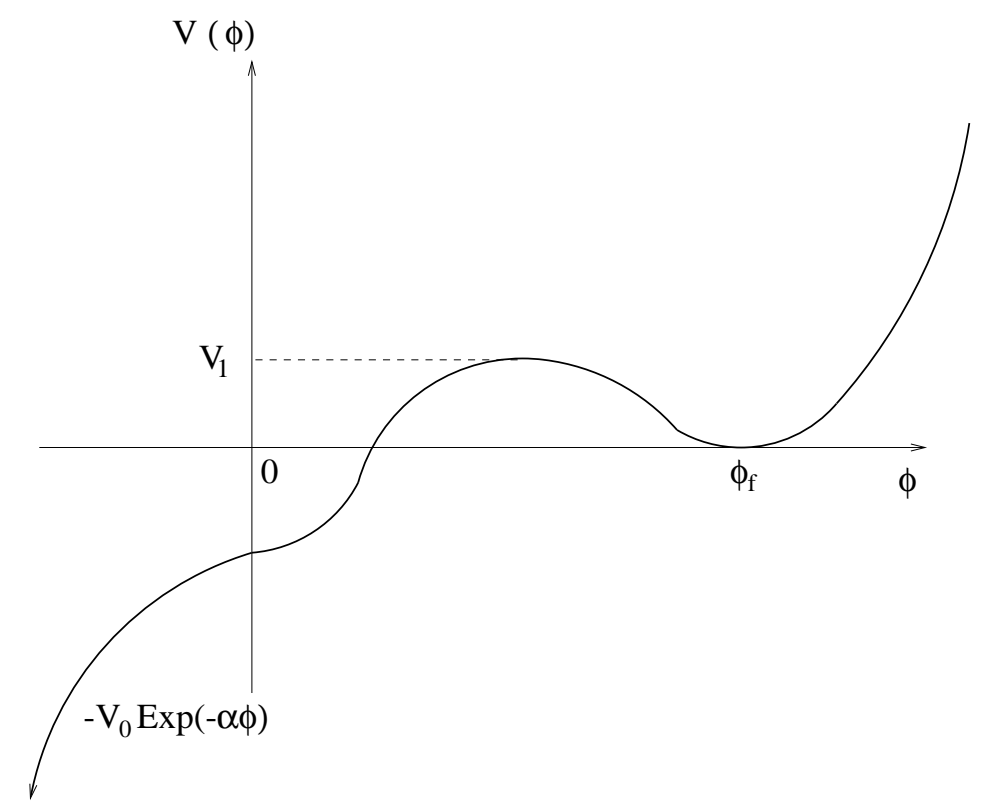

Figure 3: A potential which is unbounded from below for negative values of $\phi$.

negative $\phi$ so that it falls off exponentially $V=-V_{0} e^{-\alpha \phi}$ (see Fig. 3). Since this potential is unbounded from below, one might expect that one can always construct negative energy solutions. But this is not the case. We now show that there is a critical value, $\alpha^{2}=6$, such that if $\alpha^{2}<6$ the energy can stay positive by an order one barrier. 
As we saw above, the key point is how low can we make the energy density inside a ball of radius $R_{0}$, if we require $\phi\left(R_{0}\right)=0$. If we keep $\phi=0$ everywhere inside the ball, the energy density would be $-V_{0}$. If we include negative values of $\phi$, we can access the growing negative potential, but we have to pay the price of the derivative contributions to the energy and the exponential enhancement of the barrier. Clearly, to make the energy density as negative as possible, we want $\phi$ to change slowly. This minimizes the positive $\phi^{2}$ contribution to the energy and increases the region over which the negative potential contributes. However, the most important effect is the exponential factor. To minimize the exponential, we take $\phi=\mu \ln \left(r / R_{0}\right)$. This configuration is singular at the origin, but we will smooth it out by setting $\phi$ equal to a constant $-\phi_{0}<0$ for $r<r_{0}$. Thus $\phi_{0}=\mu \ln \left(R_{0} / r_{0}\right)$. Since $\phi$ is constant, the exponential vanishes for $r<r_{0}$ and for $r_{0}<r<R_{0}$ we have

$$
\int_{r_{0}}^{r} r \phi^{\prime 2}=\mu^{2} \ln \left(r / r_{0}\right) \text {. }
$$

Using the general solution (4.5) we get

$$
\begin{aligned}
2 m\left(R_{0}\right)= & -\frac{r_{0}^{3} V_{0}}{3}\left(\frac{r_{0}}{R_{0}}\right)^{\frac{\mu^{2}}{2}-\alpha \mu}-\frac{2 V_{0}}{\mu^{2}-2 \alpha \mu+6}\left[R_{0}^{3}-r_{0}^{3}\left(\frac{r_{0}}{R_{0}}\right)^{\frac{\mu^{2}}{2}-\alpha \mu}\right] \\
& +\frac{\mu^{2}}{\mu^{2}+2}\left[R_{0}-r_{0}\left(\frac{r_{0}}{R_{0}}\right)^{\mu^{2} / 2}\right] .
\end{aligned}
$$

The first term is the contribution to the energy from the inner region $r<r_{0}$, the second term is the contribution from the potential in the region $r_{0}<r<R_{0}$, and the last term is the contribution from the derivative terms. To make the energy density inside $R_{0}$ as negative as possible, we should choose $r_{0} \ll R_{0}$. Then

$$
\frac{m\left(R_{0}\right)}{R_{0}^{3}}=-\frac{V_{0}}{\mu^{2}-2 \alpha \mu+6} .
$$

For $\alpha^{2}<6$, this is maximized at $\mu=\alpha$, when the right hand side becomes $-V_{0} /(6-$ $\alpha^{2}$ ). If $\alpha^{2} \geq 6$, the energy density inside $R_{0}$ can be made arbitrarily negative, by choosing $\mu$ so that the denominator in (4.12) is as small as one wants. In this case, no finite positive barrier can prevent the total ADM energy from being negative. Recall that the values of $\alpha$ that arise from compactification (3.9) all satisfy $\alpha^{2}<6$.

One can show that $\phi(r)=\alpha \ln \left(r / R_{0}\right)$ is indeed an extremal path for the potential energy, given an exponential potential $V=-V_{0} e^{-\alpha \phi}$. If one changes this path by a 
small perturbation $\varepsilon(r)$ with $\varepsilon\left(R_{0}\right)=0$, then the $R_{0}^{3}$ contribution to the energy does not change to first order in $\varepsilon$.

\section{$5 \quad$ Positive energy theorems}

Despite the fact that the energy density can be arbitrarily negative, the total energy must remain positive. This follows from a simple extension of Witten's proof [3] of the positive energy theorem ${ }^{6}$. We first review the original four dimensional version, and then discuss its extension to higher dimensions. Consider a four dimensional asymptotically flat spacetime with matter satisfying the dominant energy condition: $T_{\mu \nu} t^{\mu} \tilde{t}^{\nu} \geq 0$, where $t^{\mu}$ and $\tilde{t}^{\nu}$ are any two future directed timelike vectors. Let $\Sigma$ be a nonsingular, asymptotically flat spacelike surface and let $D_{i}$ be the projection of the spacetime covariant derivative into $\Sigma$. Let $\epsilon$ be a solution of the spatial Dirac equation $\gamma^{i} D_{i} \epsilon=0$ which approaches a constant spinor $\epsilon_{0}$ at infinity. Then one can show that

$$
\oint_{\infty} \epsilon^{\dagger} D_{i} \epsilon d S^{i}=\int_{\Sigma}\left[\left(D_{i} \epsilon\right)^{\dagger}\left(D^{i} \epsilon\right)+\frac{1}{2} T_{\mu \nu} n^{\mu}\left(\epsilon^{\dagger} \gamma^{\nu} \epsilon\right)\right] d \Sigma
$$

where $n^{\mu}$ is the unit normal to $\Sigma$. The right hand side is nonnegative, and the surface integral at infinity is proportional to $P_{\mu} t_{0}^{\mu}$ where $P_{\mu}$ is the total ADM four momentum and $t_{0}^{\mu}=\epsilon_{0}^{\dagger} \gamma^{\mu} \epsilon_{0}$ is a timelike (or null) vector. This proves that the total ADM energy cannot be negative. If the total energy vanishes, $T_{\mu \nu}=0, \epsilon$ is covariantly constant, and the spacetime must be Minkowski space. The only subtlety in the proof is showing that asymptotically constant solutions to $\gamma^{i} D_{i} \epsilon=0$ exist. A plausible argument was given by Witten and a rigorous proof was given in [24].

This argument easily extends to higher dimensional spacetimes which admit spinors and have a covariantly constant spinor at infinity. The calculation which leads from $\gamma^{i} D_{i} \epsilon=0$ to (5.1) continues to hold in higher dimensions. Since supersymmetric vacuum compactifications $K$ always admit covariantly constant spinors, solutions which asymptotically approach $M_{4} \times K$ cannot have negative total energy. Furthermore, the only solution with zero energy is $M_{4} \times K$ itself. Once again, the only subtlety is the existence of appropriate solutions to the spatial Dirac equation. For CalabiYau compactifications, this has recently been shown rigorously by Dai [5]. It seems

\footnotetext{
${ }^{6}$ The proof by Schoen and Yau [23] does not extend to ten or eleven dimensions.
} 
surprising that this proof of positive total energy treats K3 and Calabi-Yau compactifications identically, even though we have seen that the latter have four dimensional regions of negative energy density and the former do not. On a time symmetric surface $\Sigma=\mathbf{R}^{\mathbf{3}} \times K$ in a vacuum solution, the proof only uses the fact that the scalar curvature of $\Sigma$ vanishes, and does not care whether there are positive scalar curvature metrics on $K$ or not. In fact, Witten's proof of positive ADM energy was given before it was even known that such metrics of positive scalar curvature exist.

From a purely four dimensional viewpoint, Calabi-Yau compactifications include fields with potentials that are unbounded from below. These certainly do not satisfy the dominant energy condition, so the usual form of the (four dimensional) positive energy theorem does not apply. It is well known that for asymptotically anti de Sitter spacetimes, one can have positive energy even with potentials which are unbounded from below $[9,10]$. It is much less known that this is also possible in asymptotically flat spacetimes. However this was shown almost twenty years ago by Boucher [4] (see also [25]). The idea is to modify the covariant derivative in Witten's spatial Dirac equation to include an arbitrary function of the fields. In the simplest case of a single scalar field, one takes $\hat{D}_{i}=D_{i}+i W(\phi) \gamma_{i}$. It then turns out that one can prove positive energy provided the potential for $\phi$ takes the form

$$
V=2 W^{\prime 2}-3 W^{2}
$$

This is similar to the form of the scalar potential in $N=1$ supergravity, in terms of the superpotential $W$, but one can do this even in nonsupersymmetric theories. This potential $V$ can be unbounded from below, but if it has a local minimum at $V=0$, then there will be asymptotically flat solutions and the positive energy theorem will hold.

We do not know the exact form of the potentials which arise in Calabi-Yau compactifications far off the moduli space. If we start with Type II string theory, we obtain four dimensional $N=2$ supergravity coupled to matter, and previous analysis has mostly focused on the massless moduli fields. The positive energy theorem can be used to say something about the shape of the potential away from the moduli space. Consider the simple conformal mode that we discussed in section 3 with exponential potential. Recall that the potential was $V=-V_{0} e^{-\alpha \phi}$, with $\alpha^{2}<6$. This applies inside the region of positive scalar curvature. Moving from this region to the moduli 
space corresponds to crossing a potential barrier of height $\sim 1 / L_{K}^{2}$, where $L_{K}$ is the size of the Calabi-Yau space. A positive energy theorem is possible only if the width of the potential is large enough. If $V_{0} \sim 1 / L_{K}^{2}$, the width must be at least of order one.

It was suggested in [26] that one could take any $V(\phi)$ and solve (5.2) for $W(\phi)$, but clearly global solutions only exist for a restricted class of $V(\phi)$. We saw in section 4 that the exponential potential $V=-V_{0} e^{-\alpha \phi}$ with $\alpha^{2} \geq 6$ always has negative energy solutions and hence a solution for $W$ cannot exist. In contrast, for $\alpha^{2}<6$, one can obtain $V$ from $W=W_{0} e^{-\alpha \phi / 2}$ with $W_{0}^{2}=2 V_{0} /\left(6-\alpha^{2}\right)$. Note that the critical value of the exponent which arose in constructing negative energy solutions is precisely the one which allows a global solution for $W$.

\section{Physical consequences of negative energy den- sity}

Even though the total energy stays positive, the existence of unbounded negative energy density may have serious consequences. In this section we discuss some of these.

\subsection{Cosmic Censorship}

The existence of negative energy density may lead to violations of cosmic censorship. That is, one might have nonsingular initial data, which evolves to singularities that are not hidden inside event horizons. As an example, consider a theory with a single scalar field and negative exponential potential $V(\phi)=-e^{\alpha \phi}$ for $\phi>0$ and a local minimum at $\phi_{f}<0$ where $V=0$. (This is similar to the example in section 4 except that the sign of $\phi$ has been changed to remove some unnecessary minus signs.) Consider time symmetric initial data consisting of a constant scalar field $\phi=\phi_{0}>0$ for $r<R_{0}$ and then $\phi$ changes continuously to the local minimum of the potential at $V=0$ at larger radius. Inside the sphere $r=R_{0}$, the energy density is a negative constant, so the three geometry has constant negative curvature. Thus the evolution inside the domain of dependence of the ball of radius $R_{0}$ looks like a $k=-1$ Robertson-Walker universe. (It is NOT anti de Sitter space, since the scalar field evolves.) Under evolution, the 
scalar field rolls down the potential and the spacetime becomes singular in finite time. Since this singularity is not the result of the usual gravitational collapse, one may not form trapped surfaces or an event horizon. This would lead to a violation of cosmic censorship.

To examine this possibility further, let us first estimate the time to the singularity. Since the metric takes the standard Robertson-Walker form, $d s^{2}=-d t^{2}+a^{2}(t) d \sigma^{2}$ where $d \sigma^{2}$ is the metric on the unit hyperboloid, the field equations are

$$
\begin{gathered}
\frac{\ddot{a}}{a}=\frac{1}{3}\left[V(\phi)-\dot{\phi}^{2}\right] \\
\ddot{\phi}+\frac{3 \dot{a}}{a} \dot{\phi}-\alpha e^{\alpha \phi}=0 .
\end{gathered}
$$

We start with $\phi=\phi_{0}$, and set $\dot{\phi}=0, \dot{a}=0$ since we want time symmetric initial data. The right hand side of (6.3) is negative, so $a$ decreases under evolution and the singularity occurs when $a=0$. Since $\phi$ increases as it rolls down the potential, the right hand side of (6.3) is always less than its initial value. Thus we can get an upper bound on the time to the singularity by solving the simple equation $\ddot{a}+a e^{\alpha \phi_{0}} / 3=0$. This yields

$$
T<\frac{\sqrt{3} \pi}{2} e^{-\alpha \phi_{0} / 2}
$$

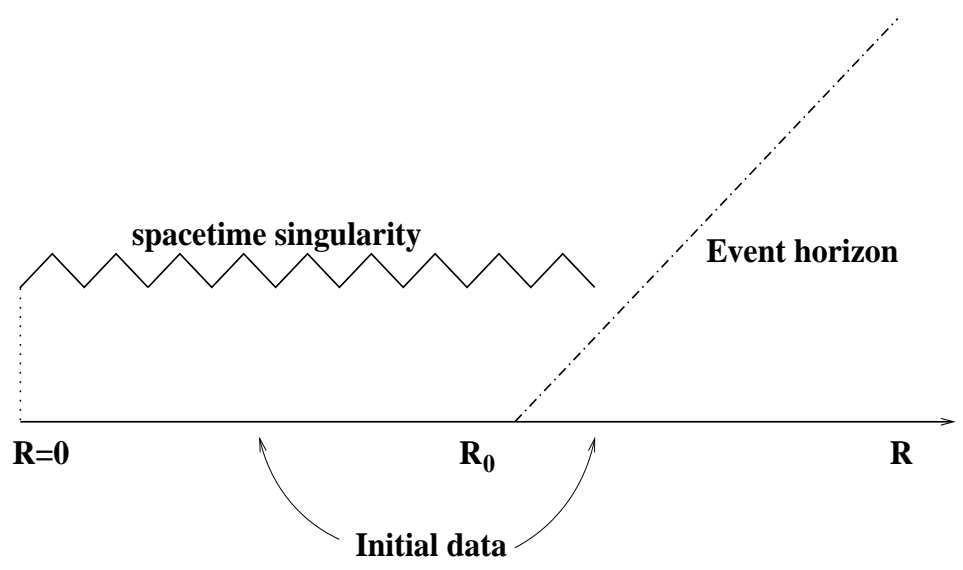

Figure 4: If an event horizon encloses the singularity, it must have an initial size of order $R_{0}$.

If we choose $R_{0} \gg T$, then the singularity will form very quickly compared to the size of the region. If the singularity lies inside a black hole, then we can trace the 
null geodesic generators of the event horizon back to the initial surface where it will form a sphere of radius approximately $R_{0}$ (see Fig. 4). Now suppose the total mass is smaller than a Schwarzschild black hole of size $R_{0}$. The area theorem for black holes only requires the weak energy condition and hence still holds even in theories with $V(\phi)<0$. Since the area of the event horizon cannot decrease during evolution and the mass cannot increase, when the spacetime settles down there is not enough mass to support a black hole large enough to enclose the singularity. Inside the domain of dependence of the initial ball of radius $R_{0}$, the singularity will be spacelike like a big crunch. The singularity is likely to extend outside the domain of dependence. There are two possibilities. Either the singularity ends, and the endpoint is a naked singularity, or the singularity continues all the way out to null infinity cutting off all spacetime. This is a disaster much worse than naked singularities.

Support for this line of thinking comes from the fact that cosmic censorship is violated in certain four dimensional field theories of this type [27]. We do not yet know if this is realized in Calabi-Yau compactifications, but it cannot be ruled out. In the scalar field example, one can show from the field equations that $\phi$ diverges at the singularity. In the Calabi-Yau context, this would correspond to $K$ shrinking to zero size. If one tries to do this keeping the metric on $K$ Ricci flat, one is likely to form black holes [28]. Since our data are time symmetric, there will be singularities in the past as well as the future. If naked singularities form in this case, it would be interesting to see if they also form in situations without singularities in the past.

Violations of cosmic censorship could be viewed as a desirable feature of CalabiYau compactifications. If singularities can be visible, one has the possibility of directly observing effects of quantum gravity. Of course, the key question is how frequently do naked singularities form. If they occur too often in certain compactifications, then those would be observationally ruled out ${ }^{7}$.

\footnotetext{
${ }^{7}$ It is not difficult to see that cosmic censorship can be violated in nonsupersymmetric compactifications, $M_{4} \times K$, if $K$ is a manifold that admits a Ricci flat metric on or close to the boundary of an $\mathcal{R}_{K}>0$ region. Of course, such theories are physically unacceptable anyway since they do not admit a positive energy theorem.
} 


\subsection{New thermal instability}

Since there is only a finite barrier separating the supersymmetric vacuum $M_{4} \times K$ from the region of unbounded negative energy density, one can ask whether ordinary finite energy processes can cause an instability. It is unlikely that a two particle collision at high energy can trigger this instability. This is because the classical process of the field going over the barrier is described quantum mechanically by a coherent state which involves many quanta. However, it is possible to see this instability in a thermal state at nonzero temperature.

At nonzero temperature, there is always an instability for nucleating a black hole [29]. There is a gravitational instanton describing this process semiclassically. The instanton is the euclidean Schwarzschild black hole with the prescribed temperature, and the nucleation rate is given by the action of this instanton. This yields $\Gamma \sim$ $e^{-M^{2}}=e^{-1 / T^{2}}$, so the nucleation of black holes is highly suppressed at temperatures much below the Planck scale $\left(T_{p l} \sim 10^{32} \mathrm{~K}\right)$.

The existence of negative energy density in Calabi-Yau compactifications means there is a new instability corresponding to thermal fluctuations causing the field to jump to the top of the barrier in some region of space and rolling over ${ }^{8}$. The height of the barrier is roughly $1 / L_{K}^{2}$ where $L_{K}$ is the size of the Calabi-Yau space. If only a small region has a thermal excitation of this amount, it just disperses. But if a region of size $L_{K}$ undergoes such a thermal excitation it will become unstable. This is because this region will now act like a horizon sized patch of de Sitter. Typically, the region briefly expands before the field rolls down the potential. If the field rolls down on the other side, the region will collapse down to a singularity [30,31]. The energy in the original thermal excitation is roughly $E \sim L_{K}$, so the decay rate is

$$
\Gamma \sim e^{-L_{K} / T} .
$$

For low temperatures $T<1 / L_{K}$, this is larger than the rate of black hole production. One could try to make this estimate more precise by looking for a new thermal instanton solution. In four dimensional gravity coupled to a scalar field with a potential as in figure 3 , the decay of the false vacuum at finite temperature would be described by an $O(3)$-symmetric gravitational instanton that is periodic in Euclidean time. In our

\footnotetext{
${ }^{8}$ If the width of the potential is small enough, this instability might manifest itself in terms of quantum tunneling through the barrier.
} 
case this would be a ten dimensional euclidean vacuum solution which asymptotically approaches $\mathbf{R}^{3} \times S^{1} \times K$. Whether or not this new thermal instability is significant in the early universe depends on the size of the internal space.

\section{Discussion}

We have reexamined the four dimensional theories resulting from supersymmetric compactifications of string theory (or just supergravity). Most previous discussions have focused on the moduli space of vacua and the associated massless fields. We have shown that if one moves off the moduli space, in many cases the four dimensional effective potentials are unbounded below. This applies to all simply connected CalabiYau and $G_{2}$ manifolds, as well as some nonsimply connected ones.

Naively, this suggests that there should be solutions with negative total ADM energy, but this is not the case. One can view the fact that the energy of all nonsingular solutions remains positive as a generalization of the phenomenon that gravity can stabilize a false vacuum [6].

We have begun an investigation of the physical effects of the negative energy density, and have discussed possible violations of cosmic censorship and new thermal instabilities. In Calabi-Yau compactifications, there is another type of possible violation of cosmic censorship In the moduli space of Ricci flat metrics, one can deform a smooth metric into a singular one, e.g., at a conifold point. Since there are solutions of the form $M_{4} \times K$ for every point on the moduli space, one may be able to change the metric on $K$ slowly enough so that no event horizon forms in the four noncompact directions even though a singularity develops on $K$. The possible violation of cosmic censorship discussed in the previous section is quite different. In our case, a singularity is forming in the four noncompact dimensions, not just on $K$. Furthermore, while it has been shown that the conifold singularity is harmless in string theory, the singularities we have been discussing may not be. However, it should be kept in mind that in neither case have violations of cosmic censorship been firmly established for Calabi-Yau compactifications.

There may be other physical consequences of the negative energy density. If these consequences are too severe, they may show that all these compactifications are

unphysical. A longstanding problem in string theory is the large number of apparently 
consistent vacua in the theory. Conceivably, the negative energy density could cut down this number dramatically.

Although we have focused on asymptotically flat solutions, the existence of potentials which are unbounded from below is likely to be important in other contexts as well $^{9}$. For example, the recent construction of de Sitter space as a solution to string theory [32] involves a Calabi-Yau internal space. Since space is now compact, there is no positive energy theorem. The negative energy density we have found provides a new type of instability of this de Sitter solution. As another example, the existence of negative energy regions surrounded by positive energy in such a way that the total energy remains positive is reminiscent of quantum field theory [33]. In the context of AdS/CFT one might wonder whether negative energy density in the (quantum) CFT is related to negative energy density in the (classical) AdS supergravity.

Just because $K 3$ does not have metrics of positive scalar curvature does not guarantee that it is free of potential instabilities. S-duality relates Type II string theory on certain Calabi-Yau manifolds to heterotic string theory on $K 3 \times T^{2}[34,35]$. In some of these cases, it is known that the Calabi-Yau space admits a metric with $\mathcal{R}_{K}>0$. The fact that the Type II string has regions of negative energy density means that the same thing must be true for the heterotic string on $K 3 \times T^{2}$. Of course the negative energy density will not arise in the same way, and may be a strong coupling effect.

Another reason for questioning the stability of $K 3$ (as well as Calabi-Yau) compactifications is some recent work of Penrose [36]. He argues that all curved compactifications should have the property that generic finite perturbations will produce curvature singularities. This is worthy of further investigation.

\section{Acknowledgments}

It is a pleasure to thank M. Cvetic, X. Dai, J. Isenberg, S. Rey, R. Roiban, J. Polchinski, I. Singer, R. Schoen, K. Schleich, K. Skenderis, M. Srednicki, J. Walcher, N. Warner, G. Wei, D. Witt, H. Verlinde, and S.T. Yau for discussions. We also thank R. Penrose for stimulating our interest in the possible instability of compact extra dimensions. This work was supported in part by NSF grant PHY-0070895 and a Yukawa fellowship.

\footnotetext{
${ }^{9}$ These applications were suggested by E. Silverstein and L. Susskind.
} 


\section{References}

[1] E. Witten, "Instability Of The Kaluza-Klein Vacuum," Nucl. Phys. B 195 (1982) 481.

[2] D. Brill and H. Pfister, "States Of Negative Total Energy In Kaluza-Klein Theory," Phys. Lett. B 228 (1989) 359; D. Brill and G. T. Horowitz, "Negative Energy In String Theory," Phys. Lett. B 262 (1991) 437.

[3] E. Witten, "A Simple Proof Of The Positive Energy Theorem," Commun. Math. Phys. 80 (1981) 381.

[4] W. Boucher, "Positive Energy Without Supersymmetry," Nucl. Phys. B 242 (1984) 282.

[5] X. Dai, "A Positive Mass Theorem for Spaces with SUSY Compactifications", to appear.

[6] S. R. Coleman and F. De Luccia, "Gravitational Effects On And Of Vacuum Decay," Phys. Rev. D 21 (1980) 3305.

[7] S. Weinberg, "Does Gravitation Resolve The Ambiguity Among Supersymmetry Vacua?," Phys. Rev. Lett. 48 (1982) 1776.

[8] M. Cvetic, S. Griffies and S. J. Rey, "Nonperturbative stability of supergravity and superstring vacua," Nucl. Phys. B 389 (1993) 3 [arXiv:hep-th/9206004].

[9] P. Breitenlohner and D. Z. Freedman, "Stability In Gauged Extended Supergravity," Annals Phys. 144 (1982) 249; "Positive Energy In Anti-De Sitter Backgrounds And Gauged Extended Supergravity," Phys. Lett. B 115 (1982) 197.

[10] G. W. Gibbons, C. M. Hull and N. P. Warner, "The Stability Of Gauged Supergravity," Nucl. Phys. B 218 (1983) 173.

[11] B. Lawson and M. Michelsohn, Spin Geometry, Princeton University Press, Princeton, NJ (1989). 
[12] R. Schoen and S. Yau, "The structure of manifolds with positive scalar curvature", Manuscripta Math. 28 (1979) 159; M. Gromov and H. Lawson, "Spin and scalar curvature in the presence of a fundamental group I", Ann of Math 111 (1980) 209.

[13] N. Hitchen, "Harmonic Spinors", Adv. in Math. 14 (1974) 1.

[14] S. Stolz, "Simply Connected Manifolds of Positive Scalar Curvature", Bull. Amer. Math. Soc. 23, 427 (1990).

[15] M. Gromov and H. Lawson, "The classification of simply connected manifolds of positive scalar curvature", Ann of Math 111 (1980) 423.

[16] W. Dwyer, T. Schick, and A. Stolz, "Remarks on a conjecture of Gromov and Lawson", math.GT/0208011.

[17] J. Rosenberg and S. Stolz, "A 'stable' version of the Gomov-Lawson conjecture", Contemp. Math 181 (1995) 405; dg-ga/9407002.

[18] B. T. McInnes, "Topologically induced instability in string theory," JHEP 0103 (2001) 031 [arXiv:hep-th/0101136].

[19] R. Schoen, "Conformal deformation of a Riemannian metric to constant scalar curvature", J. Differential Geom. 20 (1984) 479; J. Lee and T. Parker, "The Yamabe Problem", Bull. Am. Math. Soc. 17 (1987) 37.

[20] D. Witt, private communication.

[21] D. Pollack, "Nonuniqueness and High Energy Solutions for a Conformally Invariant Scalar Equation", Comm. Anal. and Geom. 1 (1993) 347.

[22] D. Pollack, private communication.

[23] R. Schoen and S. Yau, "Positivity of the Total Mass of a General Space-Time," Phys. Rev. Lett. 43 (1979) 1457.

[24] T. Parker and C. H. Taubes, "On Witten's Proof Of The Positive Energy Theorem," Commun. Math. Phys. 84 (1982) 223. 
[25] P. K. Townsend, "Positive Energy And The Scalar Potential In Higher Dimensional (Super)Gravity Theories," Phys. Lett. B 148 (1984) 55.

[26] O. DeWolfe, D. Z. Freedman, S. S. Gubser and A. Karch, "Modeling the fifth dimension with scalars and gravity," Phys. Rev. D 62 (2000) 046008 [arXiv:hepth/9909134].

[27] T. Hertog, G.T. Horowitz, K. Maeda, to appear.

[28] J. Geddes, "The collapse of large extra dimensions," Phys. Rev. D 65 (2002) 104015 [arXiv:gr-qc/0112026].

[29] D. J. Gross, M. J. Perry and L. G. Yaffe, "Instability Of Flat Space At Finite Temperature," Phys. Rev. D 25 (1982) 330.

[30] T. Banks, "T C P, Quantum Gravity, The Cosmological Constant And All That..," Nucl. Phys. B 249 (1985) 332.

[31] G. N. Felder, A. V. Frolov, L. Kofman and A. D. Linde, "Cosmology with negative potentials," Phys. Rev. D 66 (2002) 023507 [arXiv:hep-th/0202017].

[32] S. Kachru, R. Kallosh, A. Linde and S. P. Trivedi, "De Sitter vacua in string theory," arXiv:hep-th/0301240.

[33] L. H. Ford and T. A. Roman, "The quantum interest conjecture," Phys. Rev. D 60 (1999) 104018 [arXiv:gr-qc/9901074]; E. E. Flanagan, "Quantum inequalities in two dimensional curved spacetimes," Phys. Rev. D 66 (2002) 104007 [arXiv:grqc/0208066].

[34] S. Kachru and C. Vafa, "Exact results for $\mathrm{N}=2$ compactifications of heterotic strings," Nucl. Phys. B 450 (1995) 69 [arXiv:hep-th/9505105].

[35] S. Ferrara, J. A. Harvey, A. Strominger and C. Vafa, "Second quantized mirror symmetry," Phys. Lett. B 361 (1995) 59 [arXiv:hep-th/9505162].

[36] R. Penrose, "On the Instability of Extra Space Dimensions", in The Future of Theoretical Physics and Cosmology, Festschrift in honor of S.W. Hawking's 60th birthday, eds. G.W. Gibbons, S. Rankin, P. Shellard, to appear. 